\title{
Editorial: Overcoming the Challenges of Herbal Adulteration in a Globalized World
}

\author{
Anthony Booker ${ }^{1,2 *}$ \\ ${ }^{1}$ Research Group for Optimal Health, School of Life Sciences, College of Liberal Arts and Sciences, University of Westminster, \\ London, United Kingdom, ${ }^{2}$ Research Group Pharmacognosy and Phytotherapy, School of Pharmacy, University College London, \\ London, United Kingdom
}

Keywords: herbal, supply, adulterant, analytical, Sustainability , quality

Editorial on the Research Topic

Overcoming the Challenges of Herbal Adulteration in a Globalized World

The adulteration of plant based products is a global problem and one that cannot be addressed without combined efforts from industry, academia and regulators. This research topic focused on the problems of quality in the broadest sense as well as looking at specific established and new techniques to help improve the detection of sub-standard products. The issues investigated included species authentication, adulteration and contamination, value chains, agricultural practices and sustainability. Authors and reviewers contributed from Asia, the Americas and Europe, with a high percentage of published papers coming from Chinese institutions.

Of the fourteen papers that were accepted for publication, three papers focussed on documenting and highlighting the scale of the problem. Ichim and Booker in their review assessed the scale of the problem globally and found that 37 countries had significant adulteration problems. An opinion

\section{OPEN ACCESS}

Edited and reviewed by: Javier Echeverria,

University of Santiago, Chile

*Correspondence: Anthony Booker anthony.booker.11@ucl.ac.uk

Specialty section: This article was submitted to Ethnopharmacology, a section of the journal

Frontiers in Pharmacology

Received: 12 October 2021 Accepted: 03 November 2021 Published: 22 November 2021

Citation:

Booker A (2021) Editorial: Overcoming the Challenges of Herbal Adulteration in a Globalized World.

Front. Pharmacol. 12:793616. doi: 10.3389/fphar.2021.793616 piece provided by Palhares et al., the authors described the situation in Brazil and particularly in relation to sustainability and the protection of minority groups and their traditional knowledge. Luo et al. looked specifically at the on-going and increasing problem of heavy metal contamination in Chinese herbal products, something that is often affected by increased industrial pollution of the land and rivers as well as being due to cultivation or collection in areas where they are naturally occurring e.g., in cinnabar containing rock, risking mercury contamination in crops.

There were several papers that explored the value chains of medicinal plants and Kum et al. used Salvia miltiorrhiza as an example of how incorrect species might enter the supply chain and how different processing and storage techniques can affect the end quality of products. Whereas Bi et al. investigated the value chains of Astragalus membranaceus var. mongholicus and assessed the quality in relation to the geographical origin of the material and the effects of using different systems of production and suggested sustainable cultivation as a workable alternative to wild Research Topic. Cultivation may be a viable sustainable option to uncontrolled wild collection, however start up costs and lack of infrastructure can inhibit this process and more governmental and industrial support is needed for these practices to be established long-term.

Other researchers provided new ways to assess the quality of herbal products. Woodley et al. conducted a review of chemical methods and described how mitochondrial activity could be used as a new measure for assessing the biological potency of herbal products and gave detailed methodology on how this could be achieved. Ichim and De Boer described the variation in Ginseng quality and how there were other problems apart from incorrect species, including the wrong part of the plant used and incorrect age of the plant at the time of harvesting (ginseng should be at least 5 years old prior to harvesting in order to obtain the desired amounts of metabolites). There were three papers 
on shotgun metabarcoding and $\mathrm{Yu}$ et al. presented this as a powerful tool for the molecular identification of biological ingredients. This was endorsed by Liu et al. who described how adulterating fungi could be detected in complex herbal mixtures using this technique. Xie et al. showed how shotgun metabarcoding could overcome biased PCR amplification and be used to authenticate herbal mixtures with distinct variation of dosages of individual ingredients. Similarly Yu et al. described specific mini- DNA barcode techniques for use with highly processed herbal products.

Some of the new techniques (or known techniques repurposed) focussed on single plants and $\mathrm{Yu}$ et al. described how a method was developed for the analysis of Ophiopogon japonicus that incorporated quantitative microscopy. This allowed for the differentiation of quality within the same species and highlighted the concept of da di (superior quality) herbs in the Chinese herbal supply chain. Ichim et al. also looked at how microscopy could be used as a cost-effective and rapid method to identify impurities within mixtures although with the caveat that it was difficult to use with highly processed materials e.g., extracts. Zhao et al. described vector control quantitative analysis as a new method for analysing complex formulations. A PCR strategy was explained that rapidly generated the integrated DNA fragments needed from multiple targeted species. A technique with much potential for future use within the industry.

It is clear that there are global initiatives in place to improve the detection of poor quality and adulterated material in herbal medicines and there has been advances in many analytical techniques in an effort to overcome the difficulties encountered in analysing complex mixtures of medicinal plants. There appears to be growing emphasis on investigating quality problems along the length of the value chain as opposed to focussing on the finished products and with this comes an increased awareness of good collection and cultivation practices and a greater resolve to tackle issues of sustainability. Overall the papers emphasise the need for effective quality management along the length of the supply chain and the continued application of well known and new techniques to improve herbal quality and safety.

\section{AUTHOR CONTRIBUTIONS}

The author confirms being the sole contributor of this work and has approved it for publication.

Conflict of Interest: The author declares that the research was conducted in the absence of any commercial or financial relationships that could be construed as a potential conflict of interest.

Publisher's Note: All claims expressed in this article are solely those of the authors and do not necessarily represent those of their affiliated organizations, or those of the publisher, the editors and the reviewers. Any product that may be evaluated in this article, or claim that may be made by its manufacturer, is not guaranteed or endorsed by the publisher.

Copyright (C) 2021 Booker. This is an open-access article distributed under the terms of the Creative Commons Attribution License (CC BY). The use, distribution or reproduction in other forums is permitted, provided the original author(s) and the copyright owner(s) are credited and that the original publication in this journal is cited, in accordance with accepted academic practice. No use, distribution or reproduction is permitted which does not comply with these terms. 